\title{
The kinematics of intracluster planetary nebulae and the on-going subcluster merger in the Coma cluster core ${ }^{\star}$
}

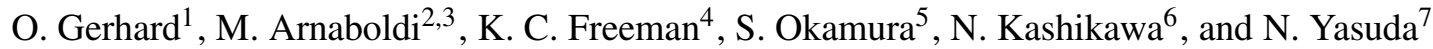 \\ 1 Max-Planck-Institut fur Extraterrestrische Physik, Giessenbachstrasse, 85741 Garching, Germany \\ e-mail: gerhard@mpe.mpg.de \\ 2 ESO, Karl-Schwarzschild-Str. 2, 85748 Garching, Germany \\ e-mail: marnabol@eso.org \\ 3 INAF, Oss. Astr. di Torino, Strada Osservatorio 20, 10025 Pino Torinese, Italy \\ 4 RSAA, Mt. Stromlo Observatory, Cotter Road, Weston Creek, ACT 2611, Australia \\ e-mail: kcf@mso.anu.edu.au \\ 5 Dept. of Astronomy and RESCEU, School of Science, The Univ. of Tokyo, Tokyo 113-0033, Japan \\ e-mail: okamura@astron.s.u-tokyo.ac.jp \\ 6 NAOJ, 2-21-1 Osawa, Mitaka, Tokyo, 181-8588, Japan \\ e-mail: kashik@zone.mtk.nao.ac.jp \\ 7 Institute for Cosmic Ray Research, Univ. of Tokyo, Kashiwa, Chiba 277-8582, Japan \\ e-mail: yasuda@icrr.u-tokyo.ac.jp
}

Received 30 September 2006 / Accepted 7 March 2007

\section{ABSTRACT}

\begin{abstract}
Aims. The Coma cluster is the richest and most compact of the nearby clusters, yet there is growing evidence that its formation is still on-going. A sensitive probe of this evolution is the dynamics of intracluster stars, which are unbound from galaxies while the cluster forms, according to cosmological simulations.

Methods. With a new multi-slit imaging spectroscopy technique pioneered at the $8.2 \mathrm{~m}$ Subaru telescope and FOCAS, we have detected and measured the line-of-sight velocities of 37 intracluster planetary nebulae associated with the diffuse stellar population of stars in the Coma cluster core, at $100 \mathrm{Mpc}$ distance.

Results. We detect clear velocity substructures within a 6 arcmin diameter field. A substructure is present at $\sim 5000 \mathrm{~km} \mathrm{~s}^{-1}$, probably from in-fall of a galaxy group, while the main intracluster stellar component is centered around $\sim 6500 \mathrm{~km} \mathrm{~s}^{-1}, \sim 700 \mathrm{~km} \mathrm{~s}^{-1}$ offset from the nearby $\mathrm{cD}$ galaxy NGC 4874. The kinematics and morphology of the intracluster stars show that the cluster core is in a highly dynamically evolving state. In combination with galaxy redshift and X-ray data this argues strongly that the cluster is currently in the midst of a subcluster merger, where the NGC 4874 subcluster core may still be self-bound, while the NGC 4889 subcluster core has probably dissolved. The NGC 4889 subcluster is likely to have fallen into Coma from the eastern A2199 filament, in a direction nearly in the plane of the sky, meeting the NGC 4874 subcluster arriving from the west. The two inner subcluster cores are presently beyond their first and second close passage, during which the elongated distribution of diffuse light has been created. We predict the kinematic signature expected in this scenario, and argue that the extended western X-ray arc recently discovered traces the arc shock generated by the collision between the two subcluster gas halos. Any preexisting cooling core region would have been heated by the subcluster collision.
\end{abstract}

Key words. ISM: planetary nebulae: general - galaxies: cluster: general - galaxies: cluster: individual: Coma cluster galaxies: evolution

\section{Introduction}

Diffuse intracluster light (ICL) has now been observed in nearby (Feldmeier et al. 2004; Mihos et al. 2005) and in intermediate redshift clusters (Zibetti et al. 2005; Krick et al. 2006). Individual intracluster stars have been detected in the Virgo and Coma clusters (Ferguson et al. 1998; Arnaboldi et al. 2003; Gerhard et al. 2005). Recent studies show that the intracluster light contains of the order of $10 \%$ of the mass in stars overall (Aguerri et al. 2005; Zibetti et al. 2005), but in cores of dense

* Based on data collected with the FOCAS spectrograph at the Subaru Telescope, which is operated by the National Astronomical Observatory of Japan, during observing run S04A-024. and rich clusters like Coma, the local ICL fraction can be as high as 40-50\% (Thuan \& Kormendy 1977; Bernstein et al. 1995).

The large scale structure of the ICL in nearby clusters is rather complex. The recent surface brightness measurements of the ICL in the Virgo cluster by Mihos et al. (2005), down to $\mu_{V}=28 \mathrm{mag} \operatorname{arcsec}^{-2}$, have shown that the ICL is made up of a wealth of diffuse features ranging from extended low surface brightness envelopes around giant ellipticals to long, thin streamers, as well as smaller scale features associated with many Virgo galaxies. A similarly deep wide-field image of the ICL in the Coma cluster is not yet available, but existing observations indicate similar complexity on a variety of scales, including tidal features like streamers (Gregg \& West 1998) or arcs (Trentham \& Mobasher 1998), extended halos around D galaxies 
(Baum et al. 1986; Adami et al. 2005a) and extended background light (Melnick et al. 1977; Thuan \& Kormendy 1977).

Morphological studies of the ICL have some limitations though: they cannot establish whether the ICL is made up by diffuse halos superposed along the LOS, but still physically bound to the galaxies they surround, or rather by stars that are free-flying in the cluster potential. This question must be answered by spectroscopic observations of the line-of-sight velocities of intracluster stars. Presently, the only tracers which allow us to measure the kinematics and dynamics of the ICL are Intracluster planetary nebulae (ICPNe), whose [OIII]5007 emission can be used both for their identification and radial velocity measurement.

By measuring the projected phase space of ICPNe we can constrain the dynamical age of the ICL component, how and when this light originated (Napolitano et al. 2003; Murante et al. 2004; Willman et al. 2004; Murante et al. 2007). Owing to the small fluxes of distant ICPNe this was so far only possible for the nearby Virgo cluster (Arnaboldi et al. 2004). With a new multi-slit imaging spectroscopy (MSIS) technique, essentially a spectroscopic blind search technique, we have now been able to measure ICPN velocities at substantially fainter fluxes. In our first application of this technique we have measured the velocity distribution of ICPNe in a field in the inner core of the Coma cluster, approximately at the peak of the X-ray emission (Gerhard et al. 2005; Arnaboldi et al. 2007).

The Coma cluster (A1656) is the richest and most compact of the nearby clusters, and has been the subject of extensive study. Originally thought of as the prototype of a rich, regular, and relaxed cluster, it is now known to contain significant small-scale and large-scale substructure in the galaxy distribution (Fitchett \& Webster 1987; Mellier et al. 1988; Biviano et al. 1996), galaxy velocity distribution (Colless \& Dunn 1996; Adami et al. 2005b), and X-ray emissivity (Briel et al. 1992; White et al. 1993; Neumann et al. 2001, 2003). Based on the recent work Coma is believed to be undergoing several accretion and merger events. Notably, the subcluster around NGC 4839 can be well distinguished in both the galaxy distribution and velocities and in the X-ray maps, and is falling into the main Coma cluster from the SW, at a velocity of $\sim 1700 \mathrm{~km} \mathrm{~s}^{-1}$ (Mellier et al. 1988; Colless \& Dunn 1996; Neumann et al. 2001). But also the cD galaxy NGC 4889 together with an associated subcluster are believed to be merging with the main Coma cluster, often associated with NGC 4874, although the details are less clear (Colless \& Dunn 1996; Adami et al. 2005b). Finally, Adami et al. (2005b) list a number of substructures, many of which may be infalling into the Coma cluster as well.

The purpose of this paper is to ask what can be learnt by combining the measurement of the ICL kinematics in our field in the Coma core with the properties of the galaxy velocity distribution and X-ray morphology. Section 2 contains a brief summary of the MSIS technique and discusses the criteria for identifying the different emission sources in the data. We distinguish between galaxy PNe and ICPNe in the field, and discuss the velocity histogram of ICPNe. The detailed procedures used for the data reduction, the catalogue of $\mathrm{PNe}$, and their spatial and magnitude-velocity distributions are given in Arnaboldi et al. (2007). In Sect. 3 we discuss the velocity distribution of the ICPNe sample in relation with the galaxy redshift distribution and some notable features in the X-ray emission in the Coma cluster core, and develop a model for the on-going merger of the NGC 4889 subcluster with the second main subcluster in Coma around NGC 4874. Finally, we give our conclusions in
Sect. 4. In what follows we assume a distance to the Coma cluster of $95 \mathrm{Mpc}$, thus $1^{\prime \prime}=460 \mathrm{pc}$.

\section{The velocity distribution of intracluster stars in a Coma core field from MSIS observations}

\subsection{The MSIS technique}

Beyond about 20-30 Mpc distance, PNe are too faint to be detectable with narrow band surveys or slitless spectroscopy their emission disappears in the sky noise in the narrow band filter. The brightest $\mathrm{PNe}$ in the Coma cluster at $100 \mathrm{Mpc}$ distance have line fluxes of $2.2 \times 10^{-18} \mathrm{erg} \mathrm{s}^{-1} \mathrm{~cm}^{-2}$, corresponding to $\sim 20$ photons per minute through an $8 \mathrm{~m}$ telescope aperture, of which $\sim 2$ will reach the detector for a typical $\sim 10 \%$ overall system efficiency. To detect such distant and faint $\mathrm{PNe}$ requires a spectroscopic blind search technique: spectroscopic, so that only the sky noise within a few $\AA$ dilutes the emission from the PN, and blind, because the positions of these faint PNe cannot be previously determined.

The Multi-Slit Imaging Spectroscopy (MSIS) technique is such a spectroscopic blind search technique. Its first application to the detection of PNe in the Coma cluster was described by Gerhard et al. (2005). The technique combines a mask of parallel multiple slits with a narrow-band filter, centered on the redshifted [OIII] $\lambda 5007 \AA$ emission line. Spectra are obtained of all $\mathrm{PNe}$ that happen to lie behind the slits. The narrow band filter limits the length of the spectra on the CCD so that many slits can be simultaneously exposed. For each set of mask exposures only a fraction of the field is surveyed; to increase the sky coverage the mask can be stepped on the sky. The technique is similar to the approach used in the search for Ly $\alpha$ emitting galaxies at very high redshifts (Stern \& Spinrad 1999; Tran et al. 2004; Martin \& Sawicki 2004).

Our pilot survey in the Coma cluster was carried out with the FOCAS spectrograph at the Subaru telescope. The instrument was used with a mask of 70 parallel slits and a narrow band filter of FWHM $60 \AA$, centered on the [OIII] $5007 \AA$ line at the redshift of the Coma cluster. The fraction of the field surveyed by a single mask is then $\sim 12 \%$. Each spectrum extends over 43 pixels on the $\mathrm{CCD}$, and the spectral resolution is $440 \mathrm{~km} \mathrm{~s}^{-1}$. As these values show, a compromise must be found in the MSIS setup between the number of slits and hence number of objects detected, and the velocity resolution and signal-to-noise $(\mathrm{S} / \mathrm{N})$ of the emission sources, which are dependent on the spectral resolution. For a detailed description of the observing technique and the signalto-noise calculations we refer to Gerhard et al. (2005).

Three mask configurations were observed for the Coma field centered at $\alpha(\mathrm{J} 2000)$ 12:59:41.784; $\delta(\mathrm{J} 2000)$ 27:53:25.388, near the centroid of the X-ray emission in the Coma cluster core, nearly coincident with the field observed by Bernstein et al. (1995) and $\sim 5$ arcmin away from the cD galaxy NGC 4874. The data reduction of the three masks observed with the MSIS technique was carried out in IRAF and is described in Arnaboldi et al. (2007). The removal of the instrumental signature was done following standard procedures for CCD reductions. The final dispersed image of the field resembles a brick wall made up of adjacent $60 \AA$ wide two-dimensional spectra; see Fig. 2 of Gerhard et al. (2005) and Fig. 1 of Arnaboldi et al. (2007). In this image point-like, monochromatic emission sources appear unresolved in both the spatial and wavelength directions, stars appear as spatially unresolved $60 \AA$ continuum spectra, and galaxies appear as extended blobs. 


\subsection{Emission sources in the pilot study MSIS field}

In the final co-added frames we look for emission line objects and classify them according to the following criteria, described in more detail in Gerhard et al. (2005) and Arnaboldi et al. (2007):

- unresolved (both in wavelength and in space) emission line objects with no continuum, which are our intracluster planetary nebulae (ICPNe) candidates;

- spatially unresolved continuum sources with unresolved or resolved line emissions. These are most likely background galaxies;

- unresolved line emissions associated with the extended continuum halos of Coma cluster galaxies in the field - such objects are compatible with being $\mathrm{PNe}$ associated with the stellar population emitting the continuum light.

In total 60 such emission line objects were detected, of which according to these criteria

- 35 PN candidates are ICPNe, with no detectable continuum flux down to $1.6 \times 10^{-20} \mathrm{erg} \mathrm{s}^{-1} \mathrm{~cm}^{-2} \AA^{-1}$, and equivalent widths ranging from $E W>110 \AA$ to $18 \AA$;

- 20 are background objects;

- 5 are PN candidates possibly associated with extended continuum halos from nearby Coma galaxies.

The identification of the objects unresolved in both position and velocities as PNe is further supported by the fact that the emission fluxes of the brightest objects are consistent with those of the brightest PNe in a population at distance $100 \mathrm{Mpc}$, and by the fact that both lines of the [OIII] doublet were seen in all four sources with sufficient flux $\geq 4.0 \times 10^{19} \mathrm{erg} \mathrm{s}^{-1} \mathrm{~cm}^{-2}$ in the [OIII] $5007 \AA$ line and at sufficiently large recession velocity $v_{\text {obs }}>7400 \mathrm{~km} \mathrm{~s}^{-1}$ that also $\lambda 4959 \AA$ is redshifted into the wavelength range probed (Gerhard et al. 2005; Arnaboldi et al. 2007). Furthermore, as we shall see below, their distribution of recession velocities is centered around the Coma cluster and is inconsistent with a population of background objects uniformly distributed in velocity. It is possible that the sample contains a remaining contamination from so far undetected, unresolved low-luminosity background emission line galaxies, but the continuum limit rules out compact HII regions such as or brighter than observed in Virgo (Gerhard et al. 2002). The final catalogue of PN candidates from the whole dataset is given in Arnaboldi et al. (2007).

\subsection{PNe in Coma galaxies}

For the PN candidates superposed on the extended continuum halos of Coma galaxies, as well as for the ICPN candidates near such halos, it is necessary to check the measured LOS velocity against that of the galaxy, in order to confirm or rule out the PN candidate-galaxy association. In the following, the IDs of the ICPN candidates refer to their entries in the catalogue in Arnaboldi et al. (2007).

- The measured LOS velocities for IPN217 and IPN224, 6440 and $6364 \mathrm{~km} \mathrm{~s}^{-1}$ respectively, are consistent with the systemic velocity of the galaxy NGC 4876 onto which they are superposed, for which $v_{\mathrm{sys}}=6678 \mathrm{~km} \mathrm{~s}^{-1}$.

- The measured LOS velocity of candidate IPN114 is $6410 \mathrm{~km} \mathrm{~s}^{-1}$, not consistent with the systemic velocity of the underlying galaxy CGCG160-235, $v_{\text {sys }}=8114 \mathrm{~km} \mathrm{~s}^{-1}$.
Similarly, for the two candidates IPN225 $\left(v_{\mathrm{LOS}}=\right.$ $\left.5861 \mathrm{~km} \mathrm{~s}^{-1}\right)$ and IPN113 $\left(v_{\mathrm{LOS}}=6719 \mathrm{~km} \mathrm{~s}^{-1}\right)$, the comparison with the systemic velocities of the nearby Coma galaxies [GMP83-3376], $v_{\text {sys }}=6815 \mathrm{~km} \mathrm{~s}^{-1}$, and [GMP83 3383], $v_{\text {sys }}=4640 \mathrm{~km} \mathrm{~s}^{-1}$, shows very large differences, of the order of $1000 \mathrm{~km} \mathrm{~s}^{-1}$. These three PN candidates are therefore not bound to the galaxies, similarly as the Virgo ICPNe discovered serendipitously along the lineof-sight to M 86 (Arnaboldi et al. 1996).

- However, close to IPN114 and CGCG160-235, one of the IPN candidates, IPN104, has $v_{\text {LOS }}=8520 \mathrm{~km} \mathrm{~s}^{-1}$ which, given the spectral resolution, is similar enough to the velocity of CGCG160-235 that IPN104 may be bound to CGCG160-235, even though it is not directly superposed on the galaxy's continuum image. Halos extended out to $70 \mathrm{kpc}$ in virial equilibrium have been detected in Virgo ellipticals via surface brightness measurements (Mihos et al. 2005) and ICPN $v_{\text {LOS }}$ measurements (Arnaboldi et al. 2004).

In what follows, we consider the PN candidates IPN114, IPN225 and IPN113 as intracluster PNe, while IPN217, IPN224, IPN104 are considered as PNe bound to Coma Galaxies. Thus the total number of ICPNe detected in our Coma field is 37.

\subsection{Velocity distribution of ICPNe}

The LOS velocity distribution of all ICPN candidates is shown in Fig. 1: this shows a clear association with the Coma cluster. The average velocity of the distribution is $6315 \mathrm{~km} \mathrm{~s}^{-1}$ and the standard deviation is $867 \mathrm{~km} \mathrm{~s}^{-1}$, but two substructures are also clearly visible: a main peak at $v_{\mathrm{LOS}} \sim 6500 \mathrm{~km} \mathrm{~s}^{-1}$ and a secondary peak at $v_{\mathrm{LOS}} \sim 5000 \mathrm{~km} \mathrm{~s}^{-1}$. The main peak in the LOS velocity distribution of PN candidates is very close to the systemic velocity of NGC $4889, v_{\text {sys }}=6495 \mathrm{~km} \mathrm{~s}^{-1}$. The secondary peak could be associated with a dissolved galaxy from one of the subgroups discussed by Adami et al. (2005b); see Sect. 3.6 below.

Figure 1 also shows the LOS velocity distribution of Coma cluster galaxies in a $6^{\prime} \times 6^{\prime}$ field centered on the MSIS pointing. Also this distribution has multiple peaks: the middle one is coincident with the $v_{\mathrm{sys}}=6495 \mathrm{~km} \mathrm{~s}^{-1}$ of NGC 4889, and two less prominent peaks are at $v_{\text {LOS }}<5000 \mathrm{~km} \mathrm{~s}^{-1}$ and $v_{\text {LOS }} \sim$ $8100 \mathrm{~km} \mathrm{~s}^{-1}$. Thus the LOS velocity distributions of ICPNe and galaxies in the field more or less correspond; according to a Kolmogorov-Smirnov test, the probability of both being drawn from the same distribution is $16 \%$.

As a consistency check, we also show in Fig. 1 the distribution of the observed emission lines of the background objects within the filter passband. For an easier comparison with the ICPN diagram, we express the $\lambda_{\text {obs }}$ in velocities, assuming that $\lambda_{0}=5007 \AA$. The distribution of point-like continuum sources with emission line is consistent with a flat distribution within the filter passband window used for the MSIS technique, and is consistent with a uniform background galaxy population ${ }^{1}$. There is no clear association with the Coma cluster here. The difference to the velocity distribution of Coma ICPNe is clear, and is one further argument in support of our source classification.

\footnotetext{
1 The apparent absence of a few objects on the blue edge of the filter bandpass is probably caused by a slight offset between the flat field image and the scientific image, which amplifies the noise on this side of the spectrum.
} 

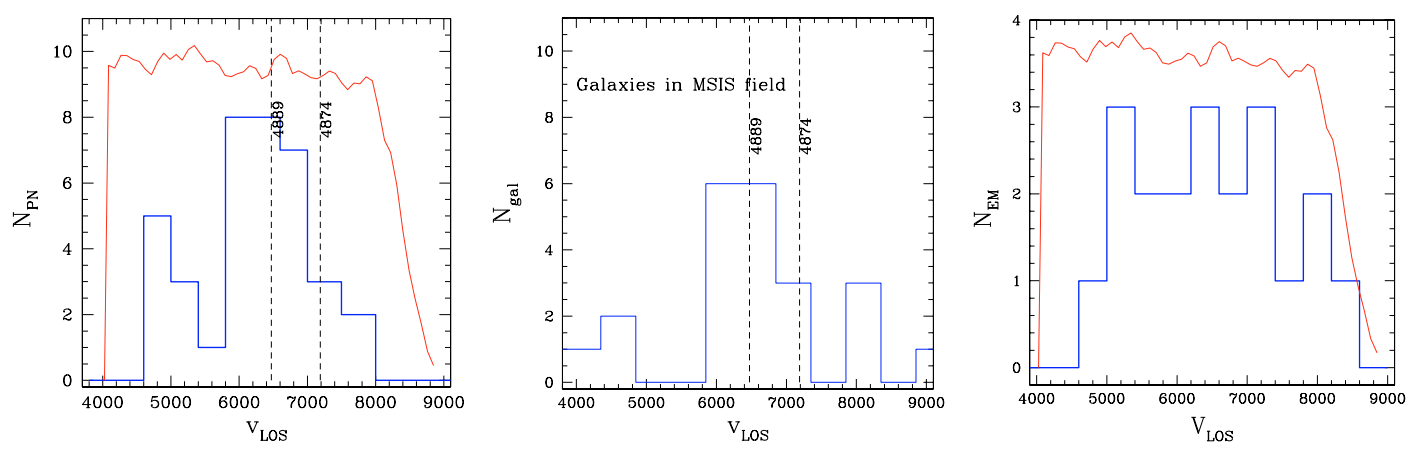

Fig. 1. LOS velocities of ICPNe, Coma galaxies, and background emission galaxies in the MSIS field. Left: histogram of the LOS velocities of ICPNe in our Coma field. The thin red line shows spectra of a star as measured through the MSIS slits, giving the filter bandpass expressed in velocity. The velocities of the two supergiant galaxies NGC 4889 and NGC 4874 in the Coma core are indicated by vertical dotted lines; that of the nearer NGC 4874 is displaced from the main peak of the ICPN distribution. Center: LOS velocity histogram for the Coma galaxies in a $6^{\prime} \times 6^{\prime}$ field centered on the MSIS field, whose velocities would fall in the velocity window allowed by the MSIS observing technique. The galaxy redshifts are from Adami et al. (2005b). Right: LOS velocity distribution of the line emitters associated with spatially unresolved continuum sources: these are compatible with a uniform population of background objects. Thin red line as in the left panel.

\section{Kinematics of diffuse light and the subcluster merger in the Coma cluster}

How does our measurement of the intracluster light kinematics in the Coma cluster core impact on the dynamical understanding of this densest and richest of the nearby clusters?

Optical and X-ray data have shown that the Coma cluster contains major substructures around the infalling giant galaxy NGC 4839 as well as around the central pair of supergiant galaxies NGC 4874 and NGC 4889 (Fitchett \& Webster 1987; Colless \& Dunn 1996; Neumann et al. 2003). In addition, a variety of smaller substructures have been identified in position-radial velocity data (Colless \& Dunn 1996; Adami et al. 2005b). These findings, combined with the modern understanding of structure formation in the hierarchical Universe, have lead to the view that the cluster continues to accrete groups from the surrounding large-scale structure, and possibly has recently had a larger accretion of a subcluster centered around NGC 4889. The other supergiant galaxy in the core, NGC 4874, is often regarded as the true nucleus of the Coma cluster in these scenarios. This is based on the fact that NGC 4874 is surrounded by the largest concentration in the projected distribution of galaxies (Biviano et al. 1996), as well as to some extent on the fact that it has a relatively large diffuse light halo. The fact that its radial velocity is significantly offset relative to the velocities of nearby galaxies is then attributed to the interaction with NGC 4889, which could have perturbed NGC 4874 out of the cluster core (Colless \& Dunn 1996).

\subsection{The intracluster light}

How does the velocity distribution of ICPNe measured in our MSIS field some $\sim 130 \mathrm{kpc}$ from NGC 4874 fit into this picture? And how can we use it to derive some more specific constraints on the merger history of the Coma cluster? The main peak of this velocity distribution is centered not at the systemic velocity of NGC 4874 at $v_{\mathrm{LOS}}=7224 \mathrm{~km} \mathrm{~s}^{-1}$, but around $v_{\mathrm{LOS}}=6500 \mathrm{~km} \mathrm{~s}^{-1}$, some $700 \mathrm{~km} \mathrm{~s}^{-1}$ off and exactly at the systemic velocity of the other, more distant supergiant galaxy NGC 4889. Clearly, these ICPNe are not bound to NGC 4874 itself, the velocity dispersion of this cD galaxy is $284 \mathrm{~km} \mathrm{~s}^{-1}$ (Smith et al. 2000), although they might be bound to the subcluster core around NGC 4874 if this is still present.

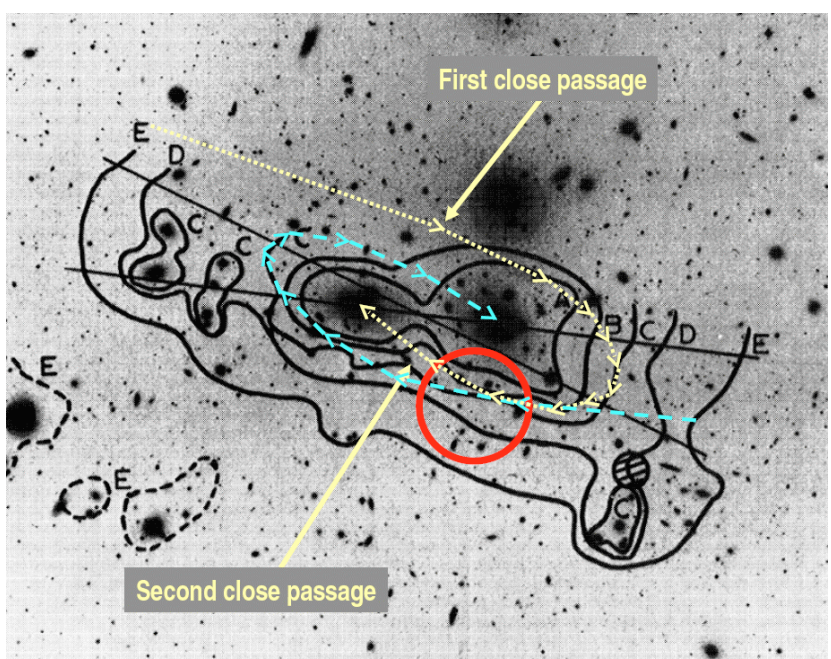

Fig. 2. The position of our MSIS field (red circle) on the diffuse light isodensity contours drawn by Thuan \& Kormendy (1977) in the Coma cluster core. The MSIS field is about $5^{\prime}$ south of NGC 4874. The second Coma cD galaxy NGC 4889 is $7^{\prime}$ east (to the left) of NGC 4874. The bright object north of NGC 4874 is the star which prevented Thuan \& Kormendy (1977) from reliably determining the northern parts of the isodensity curves in their photographic photometry. Note the strong elongation of the distribution of ICL in the Coma cluster core. The likely orbits of NGC 4889 and NGC 4874 up to their present positions are sketched by the yellow dotted and magenta dashed lines, respectively; see Sect. 3.5.

Figure 2 shows the position of our MSIS field relative to the two supergiant galaxies and the isodensity contours of ICL drawn by Thuan \& Kormendy (1977). These early ICL measurements were photographic, but agreed well with previous photoelectric measurements by Melnick et al. (1977). The contours are well-defined south of the two cluster dominant galaxies, while there are significant uncertainties on the northern side due to stray light from a bright star. In projection both galaxies appear to be embedded in a common envelope of diffuse light; as Thuan \& Kormendy state, the light is not associated with any particular galaxy. The large elongation of this diffuse light distribution together with our kinematic measurement make a strong case that this ICL in the core of Coma is in a highly dynamically evolving state - we clearly do not see a relaxed ellipsoidal distribution of IC stars here. Moreover, the paucity of diffuse light 


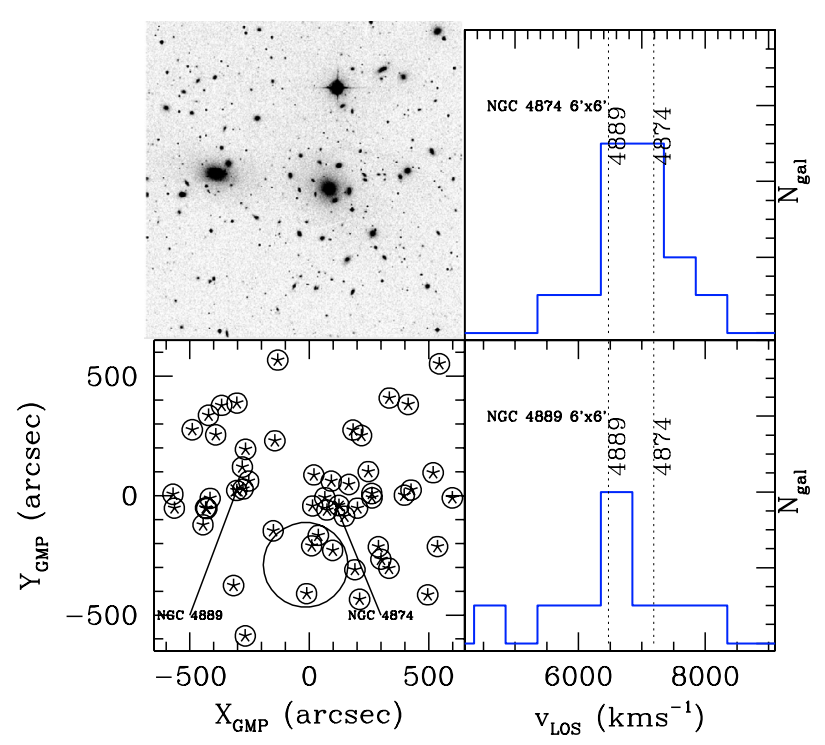

Fig. 3. The two subcluster cores around NGC 4874 and NGC 4889. Top-left: DSS image of the Coma cluster center. Bottom-left: positions of the galaxies in this region brighter than $V=17$; these are concentrated around NGC 4874 and NGC 4889. Right: velocity distributions for these bright galaxies that fall within a 36 square arcmin region around NGC 4874 (top) and NGC 4889 (bottom).

stars in this field with NGC 4874 velocities shows that the ICL in this area is poorly mixed. Mixing is expected to change the velocity distribution over a typical orbital time-scale, so the ICL in this area must have been released very recently.

\subsection{The subcluster cores}

Then the galaxies and subcluster cores from which the ICL is likely to derive will be in a state of strong interaction as well. Korchagin et al. (2001) consider a head-on collision of the two cD galaxies and suggest that this could give rise to the elongated ICL distribution, as could a collision with massive cluster substructures. Another illustration is the early binary model of Valtonen \& Byrd (1979), who estimated the masses of the subcluster cores around NGC 4874 and NGC 4889 and attempted to understand the dynamics of the Coma cluster in terms of the effects of this binary on the surrounding galaxies. Later work by Fitchett \& Webster (1987) and Biviano et al. (1996) has confirmed the existence of subclumps in the galaxy distribution centered on the two supergiant galaxies. The two dominant galaxies are projected onto the two mean peaks of the galaxy distribution. However, contrary to naive expectation, their velocities do not match the mean velocities of the peaks they are projected onto. As Colless \& Dunn (1996) state: "the positions of the peaks and the dominant galaxies appear to be almost mirror-symmetric in velocity".

However, this may be caused to considerable extent by the projection of foreground and background galaxies onto the subcluster cores. Such projection effects can be reduced by concentrating only on the brightest galaxies, which are likely to be physically closer to the dominant galaxies NGC 4874 and NGC 4889 (Valtonen \& Byrd 1979; Biviano et al. 1996). The distributions and kinematics of the bright galaxies around the two supergiant galaxies are illustrated in Fig. 3, which is based on the catalogue of Biviano et al. (1996). The top-left part of the figure shows a DSS image of the cluster center. In the

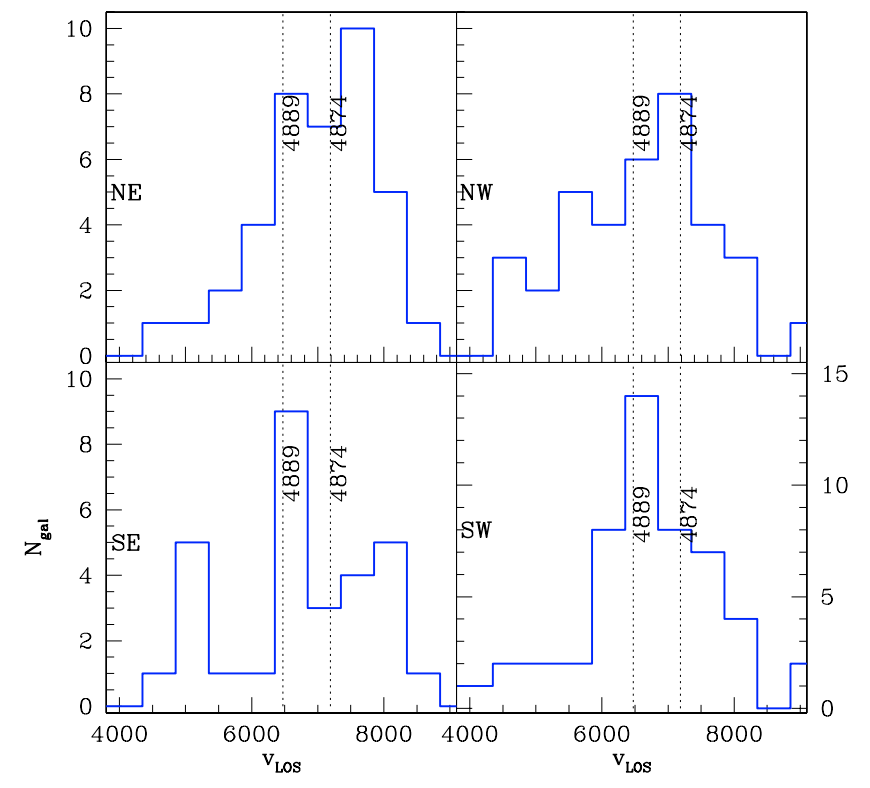

Fig. 4. The $v_{\mathrm{LOS}}$ distributions of Coma galaxies in 4 quadrants relative to the GMP83 Coma cluster center. Given in arcsec intervals along the -RA and DEC directions, NE is [-600:0, 0:600], NW is [0:600, 0:600], SE is [-600:0, -600:0], SW is [0:600, -600:0]. In GMP83 coordinates NGC 4889 is at $[-304,22]$, i.e., in the NE quadrant, and NGC 4874 is at $[124,-41]$, i.e., in the SW quadrant. The MSIS field is in the SE, at $[-15,-289]$.

bottom-left panel we show the positions of the galaxies brighter than $V=17$ in the region. Their concentration around NGC 4874 and NGC 4889 is apparent; a more quantitative analysis is in the references given above and will not be pursued here. The remaining two panels show the velocity distributions of these bright galaxies that fall in 6 arcmin squared regions around NGC 4874 and NGC 4889, respectively. The two histograms show an interesting difference: while the distribution of velocities around NGC 4874 is compact and may indicate a still selfbound cluster core, that around NGC 4889 is clearly not compact and has a twice larger velocity width. The NGC 4889 subcluster core may thus currently be dissolving, consistent with the fact that the diffuse light in our MSIS field $8^{\prime}$ west of NGC 4889 may also have been tidally dissolved from the surroundings of NGC 4889, based on its similar radial velocity.

\subsection{The NGC 4889 subcluster orbit}

Figure 4 shows the radial velocity histograms for all Coma galaxies from the catalogue of Adami et al. (2005b), in four quadrants of size $(10 \text { arcmin })^{2} \mathrm{NE}, \mathrm{NW}, \mathrm{SW}$, SE of the cluster center defined by Godwin et al. (1983, hereafter GMP83). The strong variations from one histogram to the next show that the whole Coma cluster core is far from complete virialization. Note, for example, the strong peaks in the SE quadrant at $v_{\text {LOS }}=5000 \mathrm{~km} \mathrm{~s}^{-1}$ and at $6500 \mathrm{~km} \mathrm{~s}^{-1}$; our MSIS field lies in the SE/SW quadrants (Fig. 3). However, the real surprise of Fig. 4 is that it shows clear velocity peaks around the LOS velocity of NGC 4889 in the SE and SW quadrants, with a further less significant peak in the NE quadrant, and clear peaks around the velocity of NGC 4874 in the NE and NW quadrants. A similar diagram showing the velocity histograms in $(20 \mathrm{arcmin})^{2}$ sized quadrants shows a clear peak in the NE and a very strong peak in the SW around the NGC 4889 velocity, of which the SW peak is also robust against excluding the galaxies in the cluster 
center. Also, this diagram shows robust peaks around the NGC 4874 systemic velocity in the NE and NW.

This is contrary to expectation because in a general subcluster merger, these velocity distributions should have been changed and shifted by the gravitational pull during the interaction. The only reasonable explanation for the histograms in Fig. 4 is that the average orbital accelerations on the galaxies of the two interacting subclusters on scales of $\sim 500 \mathrm{kpc}$ have predominantly been in the plane of the sky. Obviously this is a lucky coincidence; for a general orbit, the line-of-sight component of the acceleration would be visible in the distribution of galaxy recession velocities. For example, in a head-on collision along the line-of-sight, the observed galaxy velocities at the time of strong interaction would have been modified most in the interacting cluster cores and least in the subcluster outskirts. Then we should see a concentration of galaxies around the systemic velocities of NGC 4889 and NGC 4874 only in the cluster center, but not on larger scales.

The idea that the two subclusters are meeting on an orbit mostly in the plane of the sky is supported by two further arguments. First, the LOS velocity difference between the two supergiant galaxies and their surrounding cluster cores is only $\simeq 700 \mathrm{~km} \mathrm{~s}^{-1}$, while the orbital collision velocity must exceed $\sim 2000 \mathrm{~km} \mathrm{~s}^{-1}$. To estimate this we have computed the virial velocity of the Coma cluster $v_{200} \simeq 1500 \mathrm{~km} \mathrm{~s}^{-1}$ from the virial masses and radii determined by Geller et al. (1999); Lokas \& Mamon (2003). The collision velocity when the cluster cores meet will be significantly larger than the median subhalo velocity found by Hayashi \& White (2006) from simulations, $1.1 v_{200}$, because this is determined for a distribution of subhalo orbital phases. If we take the relative velocity reached by $10 \%$ of their subhalos, this gives $1.55 v_{200} \simeq 2300 \mathrm{~km} \mathrm{~s}^{-1}$. Second, the Coma cluster is part of the "Great Wall" (Geller \& Huchra 1989), a large-scale structure of clusters and filaments that extends approximately perpendicularly to the line-of-sight. In this structure Coma is connected through filaments to neighbouring large clusters, notably A2199, A1367, and A779, at approximate position angles from North through East of $80^{\circ}, 250^{\circ}$, and $275^{\circ}$ (see Fig. 3 of Adami et al. 2005b). E.g., the subcluster around the cD galaxy NGC 4839 appears to be infalling towards the main Coma cluster from the filament connecting Coma to A1367, at an estimated angle with respect to the line-of-sight of $74^{\circ}$ and velocity in the plane of the sky of $\sim 1750 \mathrm{~km} \mathrm{~s}^{-1}$ (Colless \& Dunn 1996; Neumann et al. 2001).

The direction of infall of the NGC 4889 subcluster similarly is likely to have been from one of the filaments that connect the Coma cluster with the great wall - but from which of the three filaments did it come? Biviano et al. (1996) construct a smoothed map of mean recession velocity $v_{\text {LOS }}$ for the fainter galaxies in their Coma sample. These galaxies are presumably further from the main site of gravitational interaction near the cluster cores, and thus are better tracers of the original subcluster motion. Figures 12, 13 in Biviano et al. (1996) show an interesting gradient in the smoothed 2D velocity field along a direction $35^{\circ}$ inclined with respect to the RA axis (i.e., along PA $35^{\circ}$ resp. $215^{\circ}$ ). The mean $v_{\text {LOS }}$ of the faint galaxies is $v_{\mathrm{LOS}} \simeq 6700 \mathrm{~km} \mathrm{~s}^{-1}$ at $10^{\prime}-20^{\prime} \mathrm{SW}$ of the center and increases smoothly to above $v_{\mathrm{LOS}} \simeq 7000 \mathrm{~km} \mathrm{~s}^{-1} 10^{\prime}-20^{\prime} \mathrm{NE}$ of the center. Together with Fig. 4 we interpret this gradient in the sense that the NGC 4889 subcluster now dominates WSW of the center, while the NGC 4874 subcluster is more prominent ENE of the center. Based on the elongated ICL morphology, strong interaction has already taken place between the cluster cores, so that they must have already passed through each other. Then the observed gradient requires that the NGC 4889 subcluster has last moved from ENE (the direction of A2199) across the center towards WSW, whereas the NGC 4874 subcluster must have fallen in from the West (the direction of A1367 or perhaps A779).

\subsection{Comparison with $X$-ray data and cluster simulations}

This conclusion finds independent confirmation from the recent X-ray analysis of Arnaud et al. (2001) and Neumann et al. (2003). These authors find a significant residual X-ray emission in their XMM-Newton data from a large arc-like region between the Coma cluster core and the NGC 4839 group, and a temperature enhancement on the side of this structure towards the cluster core. There is also a positive residual on the south-east side at a similar distance from the center, but with a cooler temperature; this may be associated with a group around NGC 4911. Hydrodynamic simulations of galaxy cluster collisions (Roettiger et al. 1997; Takizawa 1999; Ricker \& Sarazin 2001; Ritchie \& Thomas 2002) have shown that the compression and shocks generated in the collision give rise to large changes in X-ray luminosity, temperature, and emissivity contour shapes. After the collision of the cores of two similar mass clusters a strong arc-shaped shock is driven into the outer parts of the cluster's X-ray emitting envelope. The morphology of these arcs is reminiscent of the morphology of the large western X-ray residual in Coma (Arnaud et al. 2001; Neumann et al. 2003).

While simulations with idealized initial cluster models show strong, regular, and symmetric features, cluster mergers from cosmological initial conditions result in much more irregular morphologies (Rowley et al. 2004). The morphology of their cluster merger 13, about $300 \mathrm{Myr}$ after the collision of the cluster cores, is not unlike that observed for the Coma cluster. At this time, the emissivity contours are elongated along the direction of approach and the main arc-shaped shock is in the forward direction. From Fig. 3 of Rowley et al. (2004), we estimate that this merger increased the mass of the main cluster by $\sim 50 \%$. Thus a picture in which the NGC 4889 subcluster has recently fallen in from ENE and its centroid is now past the center of Coma towards WSW, appears consistent with the presence of the large western arc in the X-ray emission and the increased $\mathrm{X}$-ray temperature associated with it. For reference, the western arc shock would have reached its current radius of $\sim 30$ arcmin from the cluster center after 300 Myr with an average velocity of $\sim 2700 \mathrm{~km} \mathrm{~s}^{-1}$.

We mention that our interpretation is different from the suggestion by Neumann et al. (2003) that the western arc could be caused by the hot plasma of a subcluster that previously merged into Coma from the southwest, which was heated in the collision and deflected northwards by the main cluster hot athmosphere. In the hydrodynamic simulations, such deflections occur when the hot athmospheres collide before the two cluster cores have met. This is unlike the situation in the Coma cluster where, as seen from the southwest, the two subcluster cores around NGC 4874 and NGC 4889 are ahead of the western arc. On the other hand, the NGC 4839 subcluster to the southwest has not yet reached the main cluster: the X-ray observations (Neumann et al. 2003) show a depression in the emission between the NGC 4839 group and main Coma cluster (see also Neumann et al. 2001).

Our interpretation is also different from the scenario of Watanabe et al. (1999). Based on an earlier, somewhat different ASCA temperature map, they suggested that the NGC 4874 subcluster had merged into the cluster from the southeast. There is no filament in this direction connecting to a neighbouring cluster. Also the elongation direction of the ICL distribution and the 
velocity differences of the two $\mathrm{cD}$ galaxies with respect to their surrounding galaxy concentrations cannot be explained if both galaxies have just passed eachother along this direction.

Cluster mergers can disrupt cooling cores if enough gas from the secondary cluster can reach the core (Gómez et al. 2002), as happens particularly in head-on collisions of equal-mass clusters (Ritchie \& Thomas 2002). With the near-head-on geometry of the collision indicated by the ICL and galaxy velocity measurements, it is likely that a preexisting cooling core in the cluster would have been heated by the collision, consistent with the absence of a cooling core in the Coma cluster now.

\subsection{Second passage of the subcluster cores and predictions for diffuse light kinematics}

In cosmological simulations of structure formation, substructures usually collide and merge along highly radial orbits. In a nearly head-on collision of two extended structures, the outer envelopes of both structures merge in a slow oscillatory fashion along an essentially unchanging orbital direction. By contrast, the interaction of the cores is faster, stronger, and may involve a significant impact parameter. In this way the cluster cores can have deflected each other strongly while the motion of the outer clusters still looks apparently unperturbed (e.g., Murante et al. 2007).

This general picture is the likely explanation for the fact that NGC 4889 is apparently behind (towards the ENE) of the centroid of its subcluster along the direction of merging as deduced above. The elongated distribution of diffuse light requires that the galaxy has already undergone strong interaction in the core. Also, the IC star velocities show that significant diffuse light was lost west of its current position. All this is best explained if the current direction of motion of NGC 4889 is towards the east. I.e., the galaxy has gone around the other (NGC 4874) subcluster core in a highly eccentric orbit, moving presumably from north-east to south-west and then in a sharp turn towards south, east, and again north-east around NGC 4874. Such an orbit for NGC 4889 and a similar, symmetric orbit for NGC 4874 are sketched in Fig. 2. The diffuse light that we now see trailing the current position of NGC 4889 was probably lost during the orbit around the NGC 4874 subcluster core between the first and second close passages, as indicated on the figure. The material unbound from both galaxies shortly after their first close passage is likely to be the origin of the diffuse light lobes in the southwest and north-east. Note that little material is unbound from the colliding galaxies before the first pericentre passage; hence the absence of ICL with NGC 4874 velocities in our MSIS field.

This predicts that in future observations of diffuse light kinematics we should see NGC 4889 centered velocities west and south of NGC 4874 and towards the current position of NGC 4889, and more NGC 4874 centered velocities towards the northeast of NGC 4874. The latter assumes that also NGC 4874 and its cluster core has already been stripped of significant light, which is plausible in the model above but less certain considering the more intact nature of the NGC 4874 cluster core as discussed in Sect. 3.2.

One may ask whether a trace of the predicted ICL kinematics around the two cD galaxies can be seen in the velocities of nearby galaxies dissolved from the cluster cores. Figure 5 shows the spatial distribution of galaxies with different velocities in the central parts of the cluster. In the distribution of galaxies with velocities $\pm 300 \mathrm{~km} \mathrm{~s}^{-1}$ relative to the systemic velocity of NGC 4889, there is a hint of a filament connecting this cD galaxy with the region south of NGC 4874 , which is covered by the

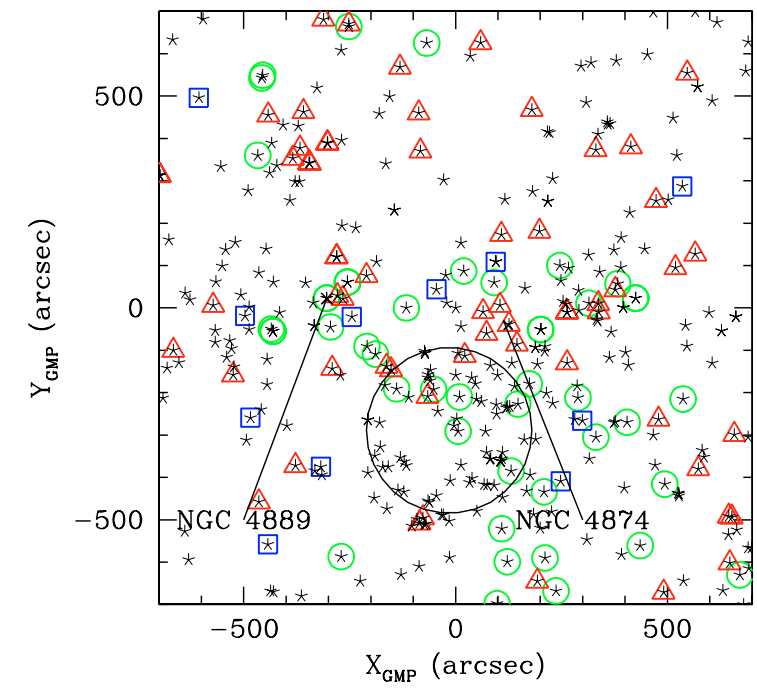

Fig. 5. Spatial distribution of all cluster members with available velocities. The $X$ and $Y$ axes denote -RA and DEC, given in arcsec relative to the GMP83 Coma cluster center, with North up and East to the left. Green circles are Coma galaxies with $6250 \mathrm{~km} \mathrm{~s}^{-1}<v_{\mathrm{LOS}}<$ $6850 \mathrm{~km} \mathrm{~s}^{-1}$, red triangles are galaxies with $7050 \mathrm{~km} \mathrm{~s}^{-1}<v_{\mathrm{LOS}}<$ $8000 \mathrm{~km} \mathrm{~s}^{-1}$, blue squares are galaxies with $4800 \mathrm{~km} \mathrm{~s}^{-1}<v_{\mathrm{LOS}}<$ $5350 \mathrm{~km} \mathrm{~s}^{-1}$. The positions of NGC 4874, NGC 4889 and the MSIS field are indicated on the plot.

MSIS field. However, the noise expected in distributions with such small numbers of galaxies precludes any more definitive conclusion.

\subsection{The $5000 \mathrm{~km} \mathrm{~s}^{-1}$ peak}

In addition to the dominant peak around NGC 4889's systemic velocity, the line-of-sight velocity distribution of ICPNe in our MSIS field contains a secondary (blue) peak at velocities around $5000 \mathrm{~km} \mathrm{~s}^{-1}$. There are also three galaxies with velocities around $4500 \mathrm{~km} \mathrm{~s}^{-1}$ in the field (Fig. 1, left and center panels, respectively). What could be the origin of these fast-moving objects in the Coma cluster core?

The list of galaxy associations in Adami et al. (2005b) contains two groups with low velocities and within less than $1 \mathrm{Mpc}$ of the MSIS field: G7 (an extended association 0.3-1.2 Mpc east of the center, with 8 members, mean velocity $5614 \mathrm{~km} \mathrm{~s}^{-1}$ ), and G9 (a compact group of 3 members $0.5 \mathrm{Mpc}$ south-west of the center, with mean velocity $5710 \mathrm{~km} \mathrm{~s}^{-1}$ ). Relative to the mean cluster velocity of $\sim 6850 \mathrm{~km} \mathrm{~s}^{-1}$ (Colless \& Dunn 1996), these galaxies have velocities of $\sim 1200 \mathrm{~km} \mathrm{~s}^{-1}$ towards the observer, while the ICPNe and galaxies in the blue peak of the MSIS field velocity distribution have even larger velocities towards us, $\sim 1600-2700 \mathrm{~km} \mathrm{~s}^{-1}$.

It is conceivable that the blue-shifted ICPNe and galaxies in the MSIS field and some or all of the galaxies in G9 and especially those in G7 are part of a single structure falling through the Coma cluster from behind. The 8 blue ICPNe would be part of the remains of a galaxy shredded by the strong tidal field in the dense cluster core, corresponding to a total stellar mass of a few times $10^{9} M_{\odot}$. This conversion assumes an $\alpha$ parameter for the first magnitude in the PN luminosity function of $\sim 10^{-8}$ (e.g., Aguerri et al. 2005), and a mass-to-light ratio of $\sim 5$. Because the MSIS field is located in the dense cluster core, the fast-moving objects in this field are probably located in a much deeper part of the Coma potential well than the galaxies in the G7 and 
G9 associations. This would explain their significantly faster line-of-sight velocities relative to the Coma cluster mean. To explain a present spread of this structure over $f \mathrm{Mpc}$ in the plane of the sky would also require differences in the velocity component perpendicular to the line-of-sight, of $\sim 1000 \mathrm{fm} \mathrm{s}^{-1}$ over the past Gyr. Thus if all the galaxies of G7 and G9 were part of this structure, it would have had to dissolve early-on during its infall into the cluster.

\section{Discussion and conclusions}

The measured offset of $\sim 700 \mathrm{~km} \mathrm{~s}^{-1}$ of the ICPNe associated with the diffuse light in the Bernstein et al. (1995) field, relative to the systemic velocity of the nearer giant elliptical (cD) galaxy NGC 4874, indicates that at least part of the diffuse light halo around NGC 4874 is not physically bound to this galaxy.

From the substructure in the galaxy velocities, the disturbed $\mathrm{X}$-ray emission, and the radio halo in the cluster, it is generally believed that the Coma cluster is in a state of on-going merging (White et al. 1993; Colless \& Dunn 1996; Neumann et al. 2003; Adami et al. 2005b, and others). Here we have shown that also the LOS velocity distribution of the ICPNe in the center of the cluster suggests an ongoing merger of two substructures in the Coma core, which is not yet virialised. The two substructures are associated with NGC 4889 and NGC 4874.

Starting with the distribution and kinematics of the diffuse light in the cluster core, we have developed a model for the geometry of this subcluster merger that is based also on the distribution of Coma galaxy velocities and the presence of structure in the X-ray emissivity and temperature distribution. In this model, the NGC 4889 subcluster fell into the region of the Coma cluster from ENE, the direction of the filament connecting Coma with A2199. Whereas the infall direction of the NGC 4874 subcluster was from the west, probably the direction of the filament towards A1367. The two subcluster cores continue to disrupt each other through their mutual gravitational interaction, with the disruption of the NGC 4889 subcluster core apparently in a more advanced stage. The inclusion of morphology and kinematics of the ICL in this model was vital because these data constrain the dynamical evolution in the cluster core itself. The ICL kinematics predicted by the model in further fields can be tested with future observations, as described in Sect. 3.5.

Currently, the two subcluster cores are past their second, close passage, during which much of the ICL seen through ICPNe must have been dissolved. This follows from the absence of any sign of mixing in the ICPN velocity distribution, and strongly supports the conclusion of Murante et al. (2007) that a large part of the ICL in clusters originates directly or indirectly from mergers of the most massive galaxies.

Several Gyr in the future, the two supergiant galaxies and their subcluster cores will have merged. With the further arrival of the NGC 4839 subcluster from the SW, the cluster may not come to rest for significantly longer. The part of the galaxy distribution centered around the NGC 4889 velocity in the 4 quadrant diagram comprises a significant fraction of all galaxies in the Coma cluster core, perhaps $30 \%$. This suggests that a truly major subcluster merger is currently taking place. Together with the other evidence for infalling substructures this suggests that Coma is forming now!

Acknowledgements. We are grateful to the on-site Subaru staff for their support. We thank C. Adami and A. Biviano for making their catalogue of galaxy velocities available in digital form.

\section{References}

Adami, C., Slezak, E., Durret, F., et al. 2005a, A\&A, 429, 39

Adami, C., Biviano, A., Durret, F., \& Mazure, A. 2005b, A\&A, 443, 17 Aguerri, J. A. L., Gerhard, O. E., Arnaboldi, M., et al. 2005, AJ, 129, 2585 Arnaboldi, M., Freeman, K. C., Mendez, R. H., et al. 1996, ApJ, 472, 145 Arnaboldi, M., Freeman, K. C., Okamura, S., et al. 2003, AJ, 125, 514 Arnaboldi, M., Gerhard, O., Aguerri, J. A. L., et al. 2004, ApJ, 614, L33 Arnaboldi, M., Gerhard, O., Okamura, S., et al. 2007, PASJ, in press Arnaud, M., Aghanim, N., Gastaud, R., et al. 2001, A\&A, 365, L67 Baum, W. A., Thomsen, B., \& Morgan, B. L. 1986, ApJ, 301, 83 Bernstein, G. M., Nichol, R. C., Tyson, J. A., Ulmer, M. P., \& Wittman, D. 1995, AJ, 110, 1507

Biviano, A., Durret, F., Gerbal, D., et al. 1996, A\&A, 311, 95

Briel, U. G., Henry, J. P., \& Böhringer, H. 1992, A\&A, 259, L31

Colless, M., \& Dunn, A. M. 1996, ApJ, 458, 435

Feldmeier, J. J., Ciardullo, R., Jacoby, G. H., \& Durrell, P. R. 2004, ApJ, 615, 196

Ferguson, H. C., Tanvir, N. R., \& von Hippel, T. 1998, Nature, 391, 461

Fitchett, M., \& Webster, R. 1987, ApJ, 317, 653

Geller, M., \& Huchra, J. P. 1989, Science, 246, 897

Geller, M., Diaferio, A., \& Kurtz, M. J. 1999, ApJ, 517, L23

Gerhard, O., Arnaboldi, M., Freeman, K. C., \& Okamura, S. 2002, ApJ, 580, L121

Gerhard, O., Arnaboldi, M., Freeman, K. C., et al. 2005, ApJ, 621, L93

Godwin, J. G., Metcalfe, N., \& Peach, J. V. 1983, MNRAS, 202, 113 (GMP83)

Gómez, P. L., Loken, C., Roettiker, K., \& Burns, J. O. 2002, ApJ, 569, 122

Gregg, M. D., \& West, M. J. 1998, Nature, 396, 549

Hayashi, E., \& White, S. D. M. 2006, MNRAS, 370, L38

Korchagin, V., Tsuchiya, T., \& Miyama, S. M. 2001, ApJ, 549, 244

Krick, J. E., Bernstein, R. A., \& Pimbblet, K. A. 2006, AJ, 131, 168

Lokas, E. L., \& Mamon, G. A. 2003, MNRAS, 343, 401

Martin, C. L., \& Sawicki, M. 2004, ApJ, 603, 414

Mellier, Y., Mathez, G., Mazure, A., et al. 1988, A\&A, 199, 67

Melnick, J., Hoessel, J., \& White, S. D. M. 1977, MNRAS, 180, 207

Mihos, C., Harding, P., Feldmeier, J., \& Morrison, H. 2005, ApJ, 631, L41

Murante, G., Arnaboldi, M., Gerhard, O., et al. 2004, ApJ, 607, L83

Murante, G., Giovalli, M., Gerhard, O., et al. 2007, MNRAS, 377, 2

Napolitano, N. R., Pannella, M., Arnaboldi, M., et al. 2003, ApJ, 594, 172

Neumann, D. M., Arnaud, M., Gastaud, R., et al. 2001, A\&A, 365, L74

Neumann, D. M., Lumb, D. H., Pratt, G. W., \& Briel, U. G. 2003, A\&A, 400, 811

Ricker, P. M., \& Sarazin, C. L. 2001, ApJ, 561, 621

Ritchie, B. W., \& Thomas, P. A. 2002, MNRAS, 329, 675

Roettiger, K., Loken, C., \& Burns, J. O. 1997, ApJS, 109, 307

Rowley, D. R., Thomas, P. A., \& Kay, S. T. 2004, MNRAS, 352, 522

Smith, R. J., Lucey, J. R., Hudson, M. J., Schlegel, D. J., \& Davies, R. L. 2000, MNRAS, 313, 469

Stern, D., \& Spinrad, H. 1999, PASP, 111, 1475

Takizawa, M. 1999, ApJ, 520, 514

Thuan, T. X., \& Kormendy, J. 1977, PASP, 89, 466

Tran, K.-V., Lilly, S. J., Crampton, D., \& Brodwin, M. 2004, ApJ, 612, L89

Trentham, N., \& Mobasher, B. 1998, MNRAS, 293, 53

Valtonen, M. J., \& Byrd, G. G. 1979, ApJ, 230, 655

Watanabe, M., Yamashita, K., Furuzawa, A., et al. 1999, ApJ, 527, 80

White, S. D. M., Briel, U. G., \& Henry, J. P. 1993, MNRAS, 261, L8

Willman, B., Governato, F., Wadsley, J., \& Quinn, T. 2004, MNRAS, 355, 159

Zibetti, S., White, S. D. M., Schneider, D. M., \& Brinkmann, J. 2005, MNRAS, 358,949 